01

\title{
Идентификация оптико-магнитных резонансов, порождаемых в разряде неона когерентными переходами пространственно разнесенных изотопически разных атомов
}

\author{
(C) Э.Г. Сапрыкин
}

Институт автоматики и электрометрии Сибирского отделения РАН, 630090 Новосибирск, Россия

e-mail: Saprykin@iae.nsk.su

Поступила в редакцию 27.08.2020 г.

В окончательной редакции 30.06.2021 г.

Принята к публикации 25.10.2021 г.

Идентифицированы инфракрасные (ИК) переходы в спектре неона в области 0.76-15.8 $\mu$ m, порождающие резонансы, обусловленные квадратурной синхронизацией полей пространственно разнесенных изотопически разных атомов. Использование численных методов анализа малоконтрастных ИК резонансов позволило оценить изотопические сдвиги уровней их родительских переходов, ранее неизвестные. Обнаружено изменение знака амплитуды квадратурных оптико-магнитных резонансов при изменении знака изотопического сдвига порождающих их переходов, и зарегистрирована зависимость времени жизни таких когерентных пар атомов от длины волны переходов, демонстрирующая необычный выход на плато при уменьшении длины волны. Предположено, что это обусловлено удлинением времени отклика возбужденных атомов окружения изотопических пар на интерференцию их полей.

Ключевые слова: неон, ИК переходы, интерференция полей, когерентные пары атомов.

DOI: $10.21883 /$ OS.2022.02.51987.223-21

\section{Введение}

Ранее было сообщено об обнаружении в излучении неонового разряда, помещенного в продольное магнитное поле с меняющейся напряженностью, необычных оптико-магнитных резонансов, смещенных относительно нуля магнитного поля [1,2]. Они возникали в смеси четных изотопов неона и не связаны с пересечением уровней сверхтонкой структуры (СТC) атомов в нечетном изотопе ${ }^{23} \mathrm{Ne}[3,4]$. Данные изотопические резонансы (ИР) появлялись при магнитных полях, компенсирующих изотопический сдвиг (ИС) линий, и получили название оптико-магнитных резонансов пересечения частот (ОМРПЧ) зеемановских компонент линий. Явление это малоизученное, и мы остановимся на механизме его возникновения, поскольку ранее в публикациях авторов его природа толковалась неоднозначно [5-10].

Изначально казалось естественным связать эффект с пересечением частот линейно поляризованных $\sigma$-компонент линий при $\Delta m=2$, описываемых выражением

$$
\left|H_{r}\right|=\frac{\Delta}{2 g_{m} \mu_{B}}
$$

где $g_{m}-$ фактор Ланде, $\mu_{B}-$ магнетон Бора, $\Delta-$ в данном случае ИС, и речь идет об интерференции полей, распространяющихся поперек направления магнитного поля. Однако идентификация ИР (определение порождающих их переходов) показала, что положение ИР в шкале магнитных полей описывается выражением c $\Delta m=1[8]^{1}:$

$$
\left|H_{r}\right|=\frac{\Delta}{g_{m} \mu_{B}} .
$$

Но для этого следует допустить возможность интерференции ортогонально поляризованных $\pi$ - и $\sigma$-компонент излучения, что в волновой оптике невозможно. Именно в связи с этим в [8] было предположено, что эффект обусловлен интерференцией квазистатических полей (КСП) перекрывающихся ближних зон изотопов, где колебания электрического поля диполей содержат как продольную, так и поперечную относительно направления магнитного поля компоненты [12]. Поэтому в этой зоне есть возможность интерференции КСП, порождающих ортогонально поляризованные волновые поля.

При выяснении природы ОМРПЧ принципиальной оказалась интерпретация наиболее контрастного сильно смещенного в шкале магнитного поля резонанса в области 1300-1400 Ое (ОМРПЧ-1350). По (1) из его смещения следуют ИС, превышающие значения, зарегистрированные в неоне. А применение (2) показало, что этот резонанс порождается переходом с уровня $2 s_{4}$ (обозначения Пашена) в основное состояние неона [11].

В [10] представлены 4 типа ОМРПЧ: синфазные, квадратурные, разностные, кооперативные резонансы, и предложена концептуально единая модель их происхождения, в которой их форма связывается с разным типом

\footnotetext{
${ }^{1}$ Отрицательные ИС приводят к отрицательным знакам магнитных полей и смене знака ОМРПЧ [11].
} 
синхронизации фаз полей излучателей. Физически она описывают нестационарную интерференцию колебаний двух частот (биение амплитуд) при ограничении времени ее регистрации (усреднения биений по времени), зависящую от разности частот $\Omega$ при разных стационарных сдвигах фаз колебаний.

Интерференция КСП разнесенных атомов изотопов начинается в результате синхронизации полей атомных диполей в перекрывающихся ближних зонах пары атомов, переводящих их в суперпозиционное когерентное состояние, и продолжается до момента скачкообразного излучения фотона с передачей атому импульса отдачи. При этом интерференция КСП пар приводит на резонансных магнитных полях к дезактивации близких к паре других возбужденных атомов с перекрывающимися зонами, преобразуя интенсивности полей интерференции пар в изменения населенности возбужденных атомов окружения, сопровождаемые уменьшением интенсивности излучения разряда вдоль $\mathrm{H}$.

В связи с этим открывается возможность получения информации о длительности интерференции полей изотопической пары (о времени жизни суперпозиционного состояния пары изотопов) и о времени подготовки отдельного атома к излучению фотона. Однако эти возможности могут быть реализованы только при знании характеристик родительских переходов: $g$-факторов уровней и длин волн, т. е. после идентификации ИР. При этом спектральная фильтрация излучения подтвердила, что когерентный процесс проявляется в виде некогерентного резонансного изменения населенности уровней. В итоге эти неравновесные изменения населенности переносятся на многие другие уровни газоразрядной плазмы, что затрудняет идентификацию ИР, но открывает дорогу к исследованию явлений, порождаемых ультрафиолетовыми (УФ) и инфракрасными (ИК) переходами за пределами спектральной чувствительности использованных приемников излучения, если идентификация осуществлена.

Ранее в работе [8] идентификация ОМРПЧ осуществлялась из визуальных оценок их положения в шкале магнитного поля. Сейчас механизм порождения резонансов стал более ясным, построены модели описания, разработаны алгоритмы программ их численного анализа, и можно продолжить работу [8], вернувшись к анализу архивных опытов и разработать процедуры для анализа новых опытов с ОМРПЧ в ИК диапазоне спектра.

\section{Моделирование резонансов тригонометрическими функциями синкуса}

Изотопические резонансы с формой центральной структуры, схожей с дисперсионным контуром, связывались с инициированием вынужденного колебания изотопического партнера с фазой, запаздывающей на $\pi / 2$. В физике такой тип интерференции не изменяет суммарную интенсивность полей в центре резонанса, поскольку при интерференции складываются квадраты амплитуд полей. Поэтому такие ИР получили название квадратурных ОМРПЧ. При их описании мы исходили из общего вида тригонометрической функции $\operatorname{sinc}(x)$ с произвольной стационарной разностью фаз колебаний полей $\Delta \varphi$ в момент времени $t=0^{2}$, отображаемой функцией $F$, которая была получена в результате усреднения по временному интервалу $T_{\text {int }}$ нестационарной интерференции двух гармонических электрических полей равных амплитуд, различающихся частотами и фазами: ${ }^{3}$

$$
\begin{gathered}
F=\frac{\sin \left(\Omega T_{\mathrm{int}}+\Delta \varphi\right)-\sin \Delta \varphi}{\Omega T_{\mathrm{int}}}, \\
\Omega=\left(\omega_{1}-\omega_{2}\right), \quad \Delta \varphi=\left(\varphi_{1}-\varphi_{2}\right) .
\end{gathered}
$$

Резонанс центрирован в нуле разности частот $\Omega$ колебаний электрических полей с частотами $\omega_{1}$ и $\omega_{2}, T_{\mathrm{int}}-$ длительность усреднения биений полей по времени их совместного существования. Контур резонанса, описываемого данной функцией в зависимости от величины $\Omega$, представляет собою резкую структуру (дисперсионную или пиковую) в нуле расщепления частот с формой, зависящей от $\Delta \varphi$. При вынужденном излучении с запаздыванием фазы на $\pi / 2$ функция $F_{q}$, описывающая квадратурный ОМРПЧ, представляет собой центральную структуру дисперсионно подобного вида:

$$
F_{q}=\frac{\sin \left(\Omega T_{\mathrm{int}}+\frac{\pi}{2}\right)-1}{\Omega T_{\mathrm{int}}}, \quad \Omega=\left(\omega_{1}-\omega_{2}\right)
$$

с примыкающими к ней при отстройке затухающими сателлитами конструктивного и деструктивного крыльев. При этом в отличие от явлений типа эффекта Дикке с $\Delta \varphi=0$, где атомы пары должны быть достаточно медленными, чтобы их относительное положение не успевало измениться за время установления их внутреннего состояния [13-15], квадратурные ОМРПЧ порождаются только движущимися относительно друг друга атомами.

Согласно опыту квадратурные ОМРПЧ порождаются при наложении резонансного магнитного поля $H_{r}$, обеспечивающего равенство разности частот излучателей $\Omega$ изотопическому сдвигу перехода, отвечающего неподвижным относительно друг друга атомам. При этом суммарная интенсивность полей не изменяется, поскольку складываются квадраты амплитуд полей. Отклонение от равенства частот приводит к невозможности вынужденного испускания и к некогерентному сложению

\footnotetext{
${ }^{2}$ Стандартная тригонометрическая синфазная функция с $\Delta \varphi=0$ для описания результатов $[1,2]$ первоначально была использована в работе [13], а затем была обнаружена наглядная демонстрация подобной синкусной формы в разряде гелия с остаточными следами смеси изотопов неона (рис. 10 в [10]). Но все последующие опыты показали, что формы резонанса со многими сателлитами это исключение, а не правило.

3 При этом амплитуды полей были вынесены из-под знака интеграла, что для ближней зоны является сильно упрощающим приближением. Но поскольку при движении атомов происходит усреднение полей, и основной вклад дают области с максимальной амплитудой полей, (3) обеспечивает хорошее согласие с опытом.
} 
интенсивностей. Поэтому резонансной особенности в окрестности совпадения частот при неподвижных атомах порождаться не должно. Но если атомы движутся в поперечном к магнитному полю направлении с относительной скоростью $\Delta v$, компенсирующей отстройку от резонанса

$$
k \delta v=\mu_{B} g\left(|H|-H_{r}\right),
$$

за счет доплеровского сдвига частоты, то резонансные условия для вынужденного испускания на магнитных полях $H \neq H_{r}$ восстанавливаются, сохраняя когерентную связь между изотопами. При этом в окрестности $H_{r}$ формируется квадратурный ОМРПЧ, в частотном представлении описываемый выражением (4). В сигнале производной он имеет экстремум на поле $H_{r}$ со знаком резонанса, зависящим от знака $\Omega$ в выражении (4), т.е. от знака ИС родительского перехода.

Но в квантовой оптике наличие квадратурной интерференции в направлении излучения исключает появление нестационарной интерференции (биений) как в этом направлении ввиду отсутствия разности излучаемых частот $\Omega$, так и в обратном направлении ввиду отсутствия излучения назад, поскольку фотоны излучаются „игольчато“. Понять причину появления биений можно, рассматривая интерференцию КСП ближних зон излучения, распространяющихся в соответствии с круговой диаграммой распределения электрического поля статического диполя. Здесь поле, инициирующее синхронизацию, распространяется не только в направлении атома, вынужденно излучающего с той же частотой, но и в обратном направлении. Равным образом и КСП вынужденно излучающего атома также распространяется в обе стороны. Однако если в прямом направлении доплеровский сдвиг, задаваемый разностью скоростей излучателей, имеющихся в среде, компенсировал разность частот, привносимую магнитным полем (отстройку от $H_{r}$ ), то в обратном направлении доплеровские сдвиги уже не компенсируют разность частот, а удваивают ее, обеспечивая необходимое для существования биений расщепление частот КСП атомов $\Omega$. При этом должен измениться и знак фазового сдвига, предсказываемый (4). ${ }^{4}$ В итоге вместо ожидаемой квадратурной функции (4) в опытах проявлялась функция с измененным знаком фазового сдвига колебаний:

$$
F_{q}=\frac{\sin \left(\Omega T_{\mathrm{int}}-\frac{\pi}{2}\right)+1}{\Omega T_{\mathrm{int}}},
$$

что в первых опытах вызывало недоумение. В (6) расщеплению частот $\Omega$ отвечает определенная относительная скорость изотопически разных атомов в плоскости, ортогональной магнитному полю, обеспечивающая компенсацию отстройки от центра резонанса. При этом каждый атом примесного изотопа находит себе партнера

\footnotetext{
${ }^{4}$ Говоря о направлении векторов скоростей изотопических атомов, напомним, что речь идет о ее проекции в плоскости, ортогональной направлению магнитного поля.
}

в распределении по скоростям атомов буферного изотопа, движущегося со скоростью, обеспечивающей компенсацию отстройки от резонанса. Однако в каждой изотопической паре разрядной кюветы с такой разностью скоростей пространственное разнесение изотопов разное, что приводит к разным амплитудам интерферирующих полей. В итоге усреднение полей пар по объему кюветы отвечает некому усредненному полю, согласующемуся с приближением, сделанным при выводе (3).

В силу реактивного характера КСП, не переносящих энергию, и их быстрого затухания обнаружить на выходе из ансамбля атомов интерференцию пар движущихся осцилляторов невозможно. В разряде она проявляется благодаря микроотклику окружающей среды на поле нестационарной интерференции пары, которое может инициировать распад возбужденных атомов близкого окружения с появлением новых некогерентных реактивных полей уже этих атомов. Последние, в свою очередь, инициируют распад следующих слоев окружающих атомов и так далее, по цепочке уменьшая их концентрацию. Для описания нелинейного характера отклика использовалась простейшая модель амплитуды отклика среды со степенным параметром $k_{s t}$ :

$$
I \sim\left(\left(1+F_{q}\right) / 2\right) k_{s t} .
$$

Увеличение отклика в конструктивном крыле квадратурного ОМРПЧ более эффективно, чем в деструктивном, и это приводит к деформации формы ИР. 5 В производной сигнала наблюдаемая пиковая форма резонанса при этом может преобразовываться в форму, приближающуюся к дисперсионной (синфазной). Как уже отмечалось ранее, сателлиты центральной структуры квадратурных ОМРПЧ были обнаружены только в кооперативных ИР, порождаемых переходами с инверсией населенностей. Основная причина подавления сателлитов это разброс времени $T_{\text {int }}$ от пары к паре изотопов вследствие разных задержек вынужденного колебания буферного изотопа относительно появления КСП, инициирующего процесс изотопа примеси. ${ }^{6}$

При переходе к циклическим частотам (MHz), которые приняты в литературе для ИС линий и преобразовании (6) в шкалу магнитных полей, использовалась функция, принимающая во внимание симметрию задачи по знаку магнитного поля (для сокращения времени счета все численные коэффициенты, включая множитель 2 у разности $\left(|H|-H_{r}\right)$, магнетон Бора, неизвестный $g$-фактор вырожденного уровня и $T_{\text {int }}$, объединены в параметр $G_{s}=1 /\left(4 \mu_{B} g_{m} T_{\mathrm{int}}\right)$, выраженный в эрстедах $(\mathrm{Oe})$ и характеризующий конкретный ОМРПЧ):

$$
F_{q}(H)=\frac{\sin \left(\left(|H|-H_{r}\right) / G_{s}-\frac{\pi}{2}\right)+1}{\left(|H|-H_{r}\right) G_{s}} .
$$

\footnotetext{
${ }^{5}$ В первых опытах за синфазные ОМРПЧ ошибочно принимали деформацию квадратурных ОМРПЧ.

6 На практике использовали процедуру нивелирования сателлитов производной теоретической функции умножением ее на обрезающий контур формы близкой к прямоугольной (см. (8) в [11]) с параметром ширины $(k k)$, выраженном в единицах ширины резонанса $\left(G_{S}\right)$.
} 
В итоге $T_{\text {int }}$ (в ns) вычислялось по результатам подгонки ширины $G_{s}$ и идентификации резонанса, дающей информацию о $g$-факторе перехода, используя выражение

$$
T_{\mathrm{int}}=1 /\left(4 \pi \mu_{B} g_{m} G_{s}\right)=226.84 / g_{m} G_{s} .
$$

\section{Эксперимент}

Первая идентификация резонансов, центрированных на 1360 и 836 Ое (ОМРПЧ1400 и ОМРПЧ-900), предполагала, что они порождаются переходами в основное состояние с уровней $1 s_{2}$ и $1 s_{4}$ [2]. Это было следствием грубости результатов [16] по оценке ИС основного состояния неона $(2 \pm 3 \mathrm{GHz})$, допускавших такой вариант. Впоследствии выяснилось, что в видимом спектре неона наблюдение ИР, порождаемых такими переходами, невозможно. Это следствие того, что их уровни распадаются непосредственно в основное состояние, минуя метастабильные состояния, аккумулирующие резонансный приход неравновесной населенности, порождаемой изотопическими парами, и увеличивающие его сравнительно с равновесной населенностью на нерезонансных магнитных полях.

Для регистрации ИР, порождаемых излучением с уровней $1 s_{2}$ и $1 s_{4}$, пришлось поставить специальные опыты с поглощением лазерного излучения с близких к ним уровней, куда неравновесная населенность напрямую переносится неупругими столкновениями (конкретно, с уровня $2 p_{4}$ [11]). Они показали малые смещения ОМРПЧ $\left(1 s_{4}-1 S_{0}\right)=200$ Ое и ОМРПЧ $\left(1 s_{2}-1 S_{0}\right)=70$ Ое, причем последний имел знак ИР, противоположный остальным наблюдаемым ОМРПЧ. Это следствие отрицательного ИС данного перехода и было первым опытом, демонстрирующим смену знака ОМРПЧ в таком процессе, что будет подтверждено далее на примере ИК переходов. Это одна из целей данной работы.

Идентификацию ОМРПЧ-1400 осуществить в [8] не удалось. На эту роль претендовали три перехода в основное состояние, но ошибка ИС последнего не позволяла сделать выбор. Причина в том, что хотя в данном случае и использовались современные оценки ИС основного состояния из работы [17], однако сами они были получены с привлечением работы [18], ограничивающей их точность. Для идентификации родительского перехода сравнивались суммарные вероятности трех переходов с этих уровней на метастабильные состояния и потом вверх. Приоритетным оказался переход $2 s_{4}-{ }^{1} S_{0}$ и, используя его, был уточнен ИС основного состояния неона [19].

В данной работе будут исследованы возможности идентификации ОМРПЧ, порождаемых ИК переходами. Ее сложность состоит в недостаточности информации об их ИС, силах осцилляторов переходов и факторах Ланде высоковозбужденных уровней. Предстоит выяснить, насколько в этих вопросах могут помочь экстраполяционные оценки, следующие из известных данных для нижележащих состояний. Поскольку речь идет о численном анализе опытов, которые уже описаны в $[6,8]$, мы повторим только ключевые характеристики опытов, дополнив их некоторыми деталями, выявившимися позднее.

Исследовалась интенсивность излучения с торца капилляра (диаметром $2.5 \mathrm{~mm}$ ) с разрядом постоянного тока, охлаждаемого водой и помещенного в продольное магнитное поле, от его напряженности, меняющейся в пределах \pm 1600 Ое. Свечение разряда регистрировалось фотодетектором, состоящим из кремниевого фотодиода и преобразователя ток-напряжение. Область спектральной чувствительности фотодетектора составляла $0.4-1.15 \mu \mathrm{m}$ с максимумом на $0.9 \mu \mathrm{m}$. Использовались инфракрасные стеклянные светофильтры (ИКС) и интерференционные фильтры.

Автоматизированная система управления сканированием магнитного поля и регистрацией сигналов обеспечивала накопление многих проходов сканирования интегрального сигнала и его производной, полученной с использованием модуляционного метода и синхронного детектирования. Переменное магнитное поле создавалось внутренним коротким соленоидом, расположенным в зоне однородного поля внешнего сканирующего соленоида. Амплитуда девиации магнитного поля частотой $450 \mathrm{~Hz}$ могла варьироваться. Для повышения стабильности разряда в области малых токов использовался слаботочный дежурный разряд, а для уменьшения уровня шумов на участки разряда, расположенные вне соленоидов, накладывался СВЧ разряд, разрушающий страты.

В опытах использовался изотоп ${ }^{20} \mathrm{Ne}$, обогащенный до $99.8 \%$, изотоп ${ }^{22} \mathrm{Ne}$ с содержанием примеси ${ }^{20} \mathrm{Ne} 7.8 \%$ и их смеси. Давление газов измерялось термопарной манометрической лампой при напуске газа в кювету с ее последующим отсечением краном и носило оценочный характер. Это следствие сорбции и десорбции газа пористым катодом с подогревом, изменяющих концентрацию изотопов в разрядном канале и соотношение изотопов, зависящее от газа, сорбированного катодом в предшествующих опытах. ${ }^{7}$

Для повышения точности информации, получаемой в опытах с ОМРПЧ, важна правильная калибровка магнитной шкалы, устанавливающая связь между током в обмотке соленоида и напряженностью магнитного поля. Эта процедура легко выполнима при условии однородности магнитного поля, одинакового для всех координат рабочего объема кюветы. В наших работах использовался соленоид большого диаметра, и магнитное поле было неоднородным. Характеристики магнитных полей, создаваемых соленоидами, сканирование и девиации полей, методика измерений эмиссионных ОМР в сигнале свечения торца газоразрядной кюветы описаны в работе [5]. Отмечено, что различие в калибровке магнитной

\footnotetext{
7 Также было возможно повышение концентрации возбужденных атомов примеси сравнительно с концентрацией в основном состоянии вследствие оптической накачки излучением буферного изотопа [20].
} 
шкалы для различных участков кюветы может приводить не только к смещению резонансов, но и к деформации их формы. Результаты последующих опытов [6,8-11] показали, что продольная пространственная неоднородность магнитных полей может приводить к резкой зависимости характеристик ОМРПЧ от параметров разряда и спектра регистрируемого излучения. Так, если регистрируется излучение сильно реабсорбированных линий, то возможна ситуация, когда сигнал определяется ближайшим к фотоприемнику концом кюветы с уменьшенным масштабом магнитной шкалы, и ОМРПЧ проявляются при больших токах соленоида. Таким образом, завышается их смещение по сравнению с ожидаемым из ИС родительских переходов. Это обстоятельство диктует необходимость регистрировать излучение на слабопоглощающих переходах между высоковозбужденными малозаселенными уровнями, где результат определяется средним магнитным полем, используя для этого ИК фильтры. Исходно коэффициент связи магнитного поля с током разряда устанавливался для его максимума в центре соленоида. В таком масштабе построены оси напряженностей магнитных полей на рисунках наших публикаций. Но при численной аппроксимации графиков, исходя из данных работы [5], с ИК фильтрами использовался корректирующий масштабирующий коэффициент $k_{m}$ перехода от максимального магнитного поля к среднему, равный 0.954.

В плане усреднения неоднородностей магнитного поля, результат, не зависящий от реабсорбции линий, должны были давать опыты с поглощением монохроматического лазерного излучения. Такой опыт был поставлен в работе [11] на переходе $3 s-2 p_{4}$ с целью уточнения ИС основного состояния неона. Это случай, когда с идентификацией резонансов вопросов не было, а точность измерений требовалось повысить, поэтому калибровке магнитного поля было уделено особое внимание. Опыты примечательны тем, что кроме ОМРПЧ, регистрируемых в видимом диапазоне спектра, были наблюдены и идентифицированы ОМРПЧ в ИК диапазоне спектра. Сравнение их смещений в шкале магнитного поля с ИС их родительских переходов привело к значению 0.955 , близкому к расчетному, и свидетельствовало об однородном пространственном распределении амплитуд ОМРПЧ, порождаемых ИК переходами. В то же время поправочный множитель для смещения доплеровских контуров на переходе $3 s-2 p_{4}$, определенный в дополнительных опытах, получил оценку $1.01 \pm 0.02$. Этот факт свидетельствовал о преобладающем вкладе в сигнал центральной части положительного столба разряда, который ранее считался однородным. Данное необычное явление связано с насыщением переходов полем собственного излучения разряда, увеличивающим населенность уровней и амплитуды ОМРПЧ в центральной части кюветы с разрядом. Позднее оно было более детально рассмотрено в [21].

\section{Результаты численного анализа опытов}

Основной физический интерес в характеристике ОМРПЧ представляют их ширины $G_{s}$. Они позволяют оценить времена нахождения пар атомов изотопов в суперпозиционных состояниях, определяемых наличием интерферирующих КСП в их перекрывающихся ближних зонах. Для осуществления этого необходимо знать $g$-факторы уровней и длины волн родительских переходов. В 2014 г. мы представили первые результаты по идентификации ИР в смеси изотопов неона [8], где апеллировали к опубликованным ИС линий неона. Тогда благодаря специфической разностной форме удалось идентифицировать ИК резонанс ОМРПЧ-350, порожденный переходом $3 p_{1}-2 s_{2}$, (рис. 5, 6 в [8]). В настоящей работе, применив численную обработку результатов опытов и оценки неизвестных ИС уровней, следующие из известных ИС линий, удалось уточнить положение и ширины еще нескольких резонансов, определить переходы, претендующие на их порождение, и обнаружить инверсию знака некоторых из них.

Результаты представляемых опытов демонстрируют рис. 1-5. На рис. 1 кружками синего цвета отмечены контуры со знаком амплитуды, отвечающим положительным ИС переходов, а красным цветом - отвечающие отрицательным ИС. Наличие ИР разного знака могло бы удивить, если бы ранее в [11] уже не было показано, что при смене знака ИС перехода резонансы меняют знак. Удивительно другое, почему ранее среди опубликованных ИС линий неона отрицательный ИС был обнаружен только у линии $2 s_{4}-{ }^{1} S_{0}$ ?

В опыте 1 (рис. 1) ОМРПЧ-90, центрированный на магнитном поле $91.8 \mathrm{Oе}$ с шириной $G_{s}=9.88 \mathrm{Oe}$,

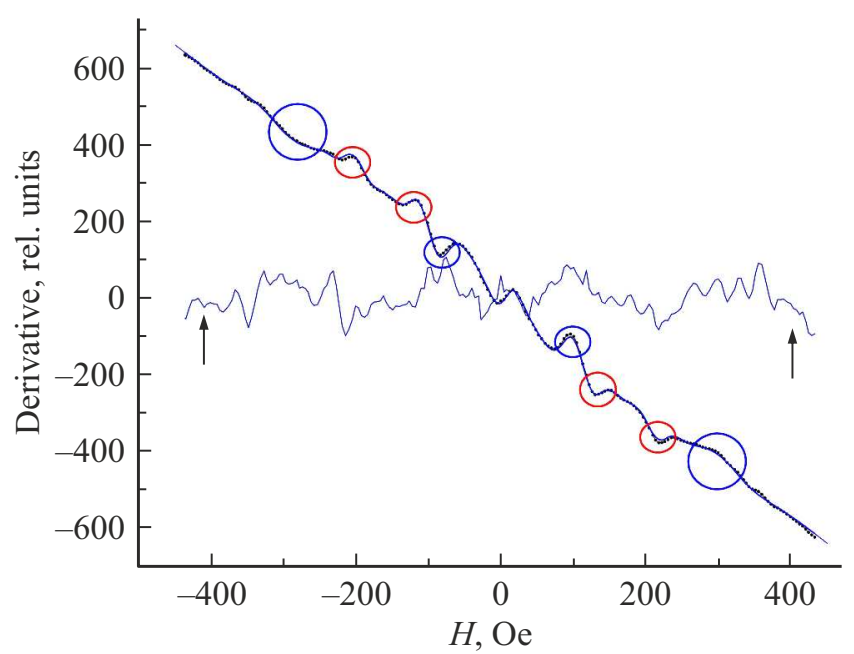

Рис. 1. Опыт 1. ОМРПЧ, центрированные на 90, 110, 200 и 280 Ое. Неон-20, $p<0.1$ Torr, $i=29 \mathrm{~mA}$, девиация $26 \mathrm{Gs}$, СФ ИКС-1, 22 прохода области сканирования. Синими кругами выделены ОМРПЧ, отвечающие положительным ИС, а красными - отвечающие отрицательным ИС. Горизонтальная кривая - увеличенные в 10 раз отклонения экспериментального графика от модельной кривой („остатки“). 
Таблица 1. Идентификация ОМРПЧ

\begin{tabular}{|c|c|c|c|c|c|c|c|c|c|c|c|c|c|c|c|}
\hline № & Опыт & ОМРПЧ & $\begin{array}{l}H_{r} \\
\text { Oe }\end{array}$ & Амплитуда & $k_{s t}$ & $\begin{array}{l}k k, \\
\text { Gs }\end{array}$ & $\begin{array}{l}G_{s}, \\
\text { Oe }\end{array}$ & $\begin{array}{c}\text { Родительский } \\
\text { переход }\end{array}$ & $\begin{array}{l}\text { ИС, } \\
\text { Ое }\end{array}$ & $\begin{array}{c}\lambda, \\
\mu \mathrm{m}\end{array}$ & $\begin{array}{l}g \text {-фак- } \\
\text { тор }\end{array}$ & $\begin{array}{l}g G \\
\text { Oe }\end{array}$ & $\begin{array}{c}T_{\text {int }}, \\
\text { ns }\end{array}$ & $\begin{array}{l}\tau, \\
\mathrm{ns}\end{array}$ & $f$ \\
\hline 1 & 2 & 3 & 4 & 5 & 6 & 7 & 8 & 9 & 10 & 11 & 12 & 13 & 14 & & 16 \\
\hline $\begin{array}{l}1 \\
2\end{array}$ & $\begin{array}{c}1 \\
3, a\end{array}$ & 90 & 91.76 & -1.1 & 1.42 & 3 & $\begin{array}{c}9.88 \\
13.48\end{array}$ & $4 d_{5}-3 p_{3}$ & 91.8 & 2.8 & 1.391 & $\begin{array}{l}13.74 \\
18.75\end{array}$ & $\begin{array}{c}16.5 \\
12.08\end{array}$ & 34 & 0.22 \\
\hline $\begin{array}{l}3 \\
4 \\
5\end{array}$ & $\begin{array}{c}1 \\
3, a \\
3, b\end{array}$ & -110 & -110 & $\begin{array}{l}0.695 \\
1.156 \\
0.147\end{array}$ & $\begin{array}{c}1 \\
1.6 \\
1.22\end{array}$ & $\begin{array}{c}3 \\
3.22 \\
3.06\end{array}$ & $\begin{array}{c}8.10 \\
9.45 \\
8.2\end{array}$ & $5 d_{5}-4 p_{3}$ & -110 & 6.17 & 1.383 & \begin{tabular}{|c|}
11.2 \\
13.07 \\
11.34
\end{tabular} & $\begin{array}{l}20.26 \\
17.34 \\
20.00\end{array}$ & 49.8 & \\
\hline $\begin{array}{l}6 \\
7 \\
\end{array}$ & $\begin{array}{l}1 \\
5\end{array}$ & -200 & $\begin{array}{c}-198.9 \\
-184\end{array}$ & $\begin{array}{c}0.51 \\
0.4 \\
\end{array}$ & $\begin{array}{l}1 \\
1\end{array}$ & $\begin{array}{l}3 \\
3\end{array}$ & $\begin{array}{l}7.89 \\
13.4 \\
\end{array}$ & $\begin{array}{l}5 s_{1}{ }^{\prime}-4 p_{1} \\
5 d_{2}-4 p_{3}\end{array}$ & $\begin{array}{l}-187 \\
-188 \\
\end{array}$ & $\begin{array}{l}5.85 \\
6.06 \\
\end{array}$ & $\begin{array}{l}0.809 \\
0.791\end{array}$ & $\begin{array}{c}6.44 \\
10.06 \\
\end{array}$ & $\begin{array}{l}35.2 \\
21.4\end{array}$ & $\begin{array}{l}96.6 \\
34.7\end{array}$ & \\
\hline $\begin{array}{c}8 \\
9 \\
10\end{array}$ & $\begin{array}{l}1 \\
2 \\
4\end{array}$ & 280 & $\begin{array}{c}276.4 \\
276 \\
276.2\end{array}$ & $\begin{array}{l}-0.94 \\
-0.37 \\
-2.34\end{array}$ & 1 & $\begin{array}{c}3 \\
10.5 \\
6.87\end{array}$ & $\begin{array}{l}17.74 \\
15.58 \\
10.76\end{array}$ & $3 p_{2}-2 s_{3}$ & 277 & 2.31 & 1.397 & $\begin{array}{l}24.78 \\
27.13 \\
15.03\end{array}$ & $\begin{array}{c}4.57 \\
10.42 \\
15.08\end{array}$ & 114 & 0.5 \\
\hline 11 & 2 & 130 & 129.3 & -1.64 & 1.08 & 10.5 & $\begin{array}{l}8.25 \\
11.0 \\
\end{array}$ & $5 p_{5}-4 s_{3}$ & 130 & 11.38 & 0.699 & $\begin{array}{l}5.77 \\
7.69\end{array}$ & $\begin{array}{l}39.3 \\
29.5 \\
\end{array}$ & 150 & \\
\hline 12 & 5 & & 129.6 & -025 & 1.03 & 3.6 & 8.5 & & & & & 5.94 & 38.18 & & \\
\hline 13 & 2 & 50 & 50.7 & 0.43 & 1.1 & 10.5 & 4.55 & & & & & & & & \\
\hline 14 & 2 & 250 & 239 & -0.24 & 1.66 & 10 & $\begin{array}{l}5.26 \\
7.46\end{array}$ & $3 d_{6}-2 p_{10}$ & 250 & 0.75 & 1.984 & $\begin{array}{c}10.43 \\
14.8\end{array}$ & $\begin{array}{c}21.72 \\
15.4\end{array}$ & 18.4 & 0.14 \\
\hline 15 & 4 & 250 & 255 & $\begin{array}{l}-0.73 \\
-0.51 \\
\end{array}$ & 1 & 3 & $\begin{array}{l}6.46 \\
9.15 \\
\end{array}$ & & & & & $\begin{array}{l}12.81 \\
18.85 \\
\end{array}$ & $\begin{array}{c}17.7 \\
12.04 \\
\end{array}$ & & \\
\hline $\begin{array}{l}16 \\
17\end{array}$ & $\begin{array}{l}3, a \\
3, b\end{array}$ & -10 & $\begin{array}{l}-11.3 \\
-7.56\end{array}$ & $\begin{array}{l}0.39 \\
0.33\end{array}$ & $\begin{array}{l}1.02 \\
1.00\end{array}$ & $\begin{array}{c}3 \\
2.85\end{array}$ & $\begin{array}{l}2.37 \\
2.14\end{array}$ & $5 s_{2}-5 p_{1}$ & $\begin{array}{l}-11.3 \\
-7.6\end{array}$ & 15.76 & 1.315 & $\begin{array}{l}3.12 \\
2.81\end{array}$ & $\begin{array}{l}72.8 \\
80.8\end{array}$ & & \\
\hline 18 & [10] & 1330 & 1330 & $\begin{array}{l}-1.69 \\
-1.37\end{array}$ & $\begin{array}{l}1.5 \\
2.6\end{array}$ & 3 & $\begin{array}{l}11 \\
21\end{array}$ & $2 s_{4}-{ }^{1} S_{0}$ & 1330 & 0.063 & 1.276 & \begin{tabular}{|c|}
6.38 \\
14.04
\end{tabular} & $\begin{array}{l}16.4 \\
8.46\end{array}$ & 10.07 & 0.012 \\
\hline
\end{tabular}

Примечание. $\tau$ - время жизни верхнего уровня родительского перехода [23], $f$ - сила осциллятора вырожденного уровня родительского перехода [23].

соседствует с ИР близкой амплитуды, но противоположного знака, центрированном на 110 Ое с шириной 8.1 Ое. Далее резонансы противоположного знака мы будем обозначать отрицательным знаком магнитного поля ОМРПЧ-(-110). Параметры ОМРПЧ и предполагаемых родительских переходов отображаются в табл. 1. В табл. 2 суммированы ожидаемые ИС уровней с $J=0,1$ (числа в скобках), могущих порождать ИР, полученные путем экстраполяции известных ИС высоковозбужденных уровней к их нулевым значениям на порогах ионизации. Жирным текстом выделены значения, полученные из литературы или при идентификации ИР.

Данные ОМРПЧ-90 опыта 1 приведены в первой строке табл. 1. Он порождается переходом $4 d_{5}-3 p_{3}$ с длиной волны $2.8 \mu \mathrm{m}$ и силой осциллятора $f=0.22$. Надежность идентификации определяется тем, что ИС состояний $4 d_{5}(106.86 \mathrm{MHz})$ и $3 p_{3}(285.6 \mathrm{MHz})$ вычислены из опубликованных данных об ИС линий. Резонанс ОМРПЧ-90 проявился также и в опыте 3 (рис. 3,a) с шириной, равной 13.48 Ое (табл. 1, строка 2). Исходя из ширин, время интерференции $T_{\text {int }}$ определилось как
(16.5-12.08) ns (табл. 1). При этом с зависимостью времени интерференции полей изотопической пары от длины волны перехода (кривая 1, рис. 6) лучше согласуется значение $12.08 \mathrm{~ns}$.

Переходом, порождающим ОМРПЧ-(-110), выбран $5 d_{5}-4 p_{3}$ с длиной волны $6.17 \mu \mathrm{m}$ (табл. 1 , строки $3-5$ ). Неизвестный ИС уровня $4 p_{3}$ был экстраполирован по ИС уровней $4 p_{9,8}$, полученным из ИС переходов на уровни $3 s_{5}$ и $3 s_{4}$, как $30 \mathrm{MHz}$, а неизвестный ИС уровня $5 d_{5}$ был определен из смещения ОМРПЧ- $(-110)$, зарегистрированного в этом опыте, как $243.04 \mathrm{MHz}$ $\left(g\left(5 d_{5}\right)=1.383\right)$. При этом он оказался близким к значениям $(238-242) \mathrm{MHz}$ уровней $5 d_{2}, 5 d_{1}{ }^{\prime \prime}, 5 s_{1}^{\prime \prime \prime \prime}$ из работы [22], если в ней исправить ошибку в знаке ИС линий переходов с этих уровней на уровни $4 p_{7,6,5}$, которые на самом деле отрицательны. Остальные 16 ИК линий этой работы были измерены правильно, поэтому найти ошибку и выявить ее причину было не просто. В итоге время жизни суперпозиционного состояния изотопической пары на длине волны $6.17 \mu \mathrm{m}$ оказалось равным 20.26 ns. То есть, как и следует, со значением, 
Таблица 2. Оценки ИС уровней неона, формирующих ОМРПЧ $(\mathrm{MHz})$

\begin{tabular}{cc|cc|cc}
\hline \multicolumn{2}{c|}{$s$-уровни } & \multicolumn{2}{c|}{$d$-уровни } & \multicolumn{2}{c}{$p$-уровни } \\
\hline $2 s_{4}(1)$ & $\mathbf{8 5 1 . 0}$ & $3 d_{6}(0)$ & $\mathbf{4 8 3 . 6 3}$ & $3 p_{10}(1)$ & 350 \\
$2 s_{2}(1)$ & $\mathbf{8 1 8 . 7 3}$ & $3 d_{5}(1)$ & $\mathbf{5 0 0 . 0}$ & $3 p_{7}(1)$ & 350 \\
$2 s_{3}(0)$ & $\mathbf{7 9 8 . 3 4}$ & $3 d_{2}(1)$ & $\mathbf{5 1 4 . 2 0}$ & $3 p_{3}(0)$ & $\mathbf{2 8 5 . 6}$ \\
$3 s_{4}(1)$ & $\mathbf{2 6 1 . 6 3}$ & $3 s_{1}{ }^{\prime}(1)$ & $\mathbf{3 9 8 . 5 8}$ & $3 p_{5}(1)$ & $\mathbf{2 5 5 . 2}$ \\
$3 s_{2}(1)$ & $\mathbf{1 7 2 . 7 3}$ & $4 d_{6}(0)$ & $\mathbf{1 0 1 . 4 7}$ & $3 p_{2}(1)$ & $\mathbf{2 5 6 . 4}$ \\
$3 s_{3}(0)$ & $\mathbf{1 4 7 . 3 3}$ & $4 d_{5}(1)$ & $\mathbf{1 0 6 . 8 6}$ & $3 p_{1}(0)$ & $\mathbf{2 2 2 . 6}$ \\
$4 s_{4}(1)$ & 102.90 & $4 d_{2}(1)$ & $\mathbf{1 2 5 . 4 3}$ & $4 p_{10}(1)$ & 30 \\
$4 s_{2}(1)$ & 103.77 & $4 s_{1}{ }^{\prime}(1)$ & $\mathbf{2 9 . 5 3}$ & $4 p_{7}(1)$ & $\mathbf{3 0}$ \\
$4 s_{3}(0)$ & $\mathbf{1 3 7 . 5 7}$ & $5 d_{6}(0)$ & $\mathbf{2 2 3 . 6 8}$ & $4 p_{3}(0)$ & $\mathbf{3 0}$ \\
$5 s_{4}(1)$ & 67.4 & $5 d_{5}(1)$ & $\mathbf{2 4 3 . 0 4}$ & $4 p_{5}(1)$ & $\mathbf{3 0}$ \\
$5 s_{2}(1)$ & $\mathbf{2 4 . 4}$ & $5 d_{2}(1)$ & $\mathbf{2 3 7 . 9 0}$ & $4 p_{2}(1)$ & 30 \\
$5 s_{3}(0)$ & 30 & $5 s_{1}{ }^{\prime}(1)$ & $\mathbf{2 4 1 . 8 0}$ & $4 p_{1}(0)$ & 30 \\
$6 s_{4}(1)$ & 10 & $6 d_{6}(1)$ & 100 & $5 p_{10}(1)$ & 20 \\
$6 s_{2}(1)$ & 10 & $6 d_{5}(1)$ & 100 & $5 p_{7}(1)$ & 20 \\
$6 s_{3}(0)$ & 10 & $6 s_{1}{ }^{\prime}(1)$ & 75 & $5 p_{3}(0)$ & 10 \\
& & & & $5 p_{5}(1)$ & $\mathbf{1 0}$ \\
& & & & $5 p_{1}(0)$ & 10 \\
& & & & $6 p_{10}(1)$ & 5 \\
& & & & $6 p_{1}(0)$ & 5
\end{tabular}

Примечание. Числа в скобках - полный угловой момент уровня.

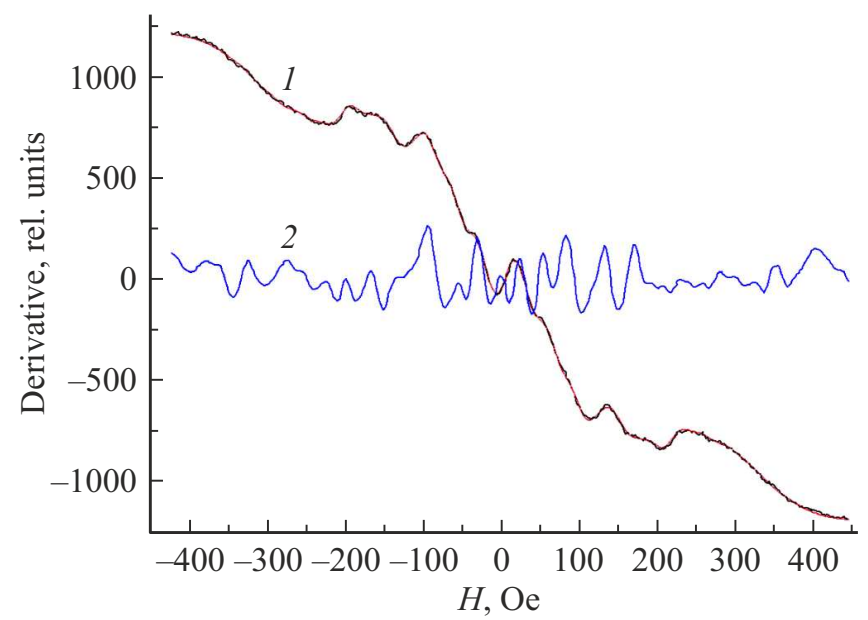

Рис. 2. Опыт 2. 1 - ОМРПЧ, центрированные на 50, 130, 200, 240 и 280 Ое. Неон-20, $p \sim 0.1$ Torr, $i=30 \mathrm{~mA}$, девиация $12 \mathrm{Gs}$, СФ ИКС-7, 3 прохода. 2 - увеличенные в 10 раз отклонения экспериментального графика от модельной кривой („остатки“).

большим, чем на переходе с длиной волны $2.8 \mu \mathrm{m}$. Этот же ОМРПЧ-(-110) проявился в опыте 3 с шириной $9.45 \mathrm{Oe}$, что приводит к $T_{\mathrm{int}}=17.34 \mathrm{~ns}$ (рис. $3, a$, табл. 1 , строка 4), и с шириной 8.2 Ое и $T_{\mathrm{int}}=20 \mathrm{~ns}$ (рис. $3, b$, табл. 1, строка 5).

Претендентом на порождение ОМРПЧ- $(-200)$ со смещением -198.9 Ое (табл. 1, строки 6,7) выступает переход $5 s_{1}{ }^{\prime}-4 p_{1}$ с длиной волны $5.85 \mu \mathrm{m}$. Здесь, если установить ИС уровня $4 p_{1}$ равным $30 \mathrm{MHz}$, как и выше, то при ИС уровня $5 s_{1}^{\prime}$, равным $242 \mathrm{MHz}$ (близким к ИС $5 d$-уровней), смещение ОМРПЧ при
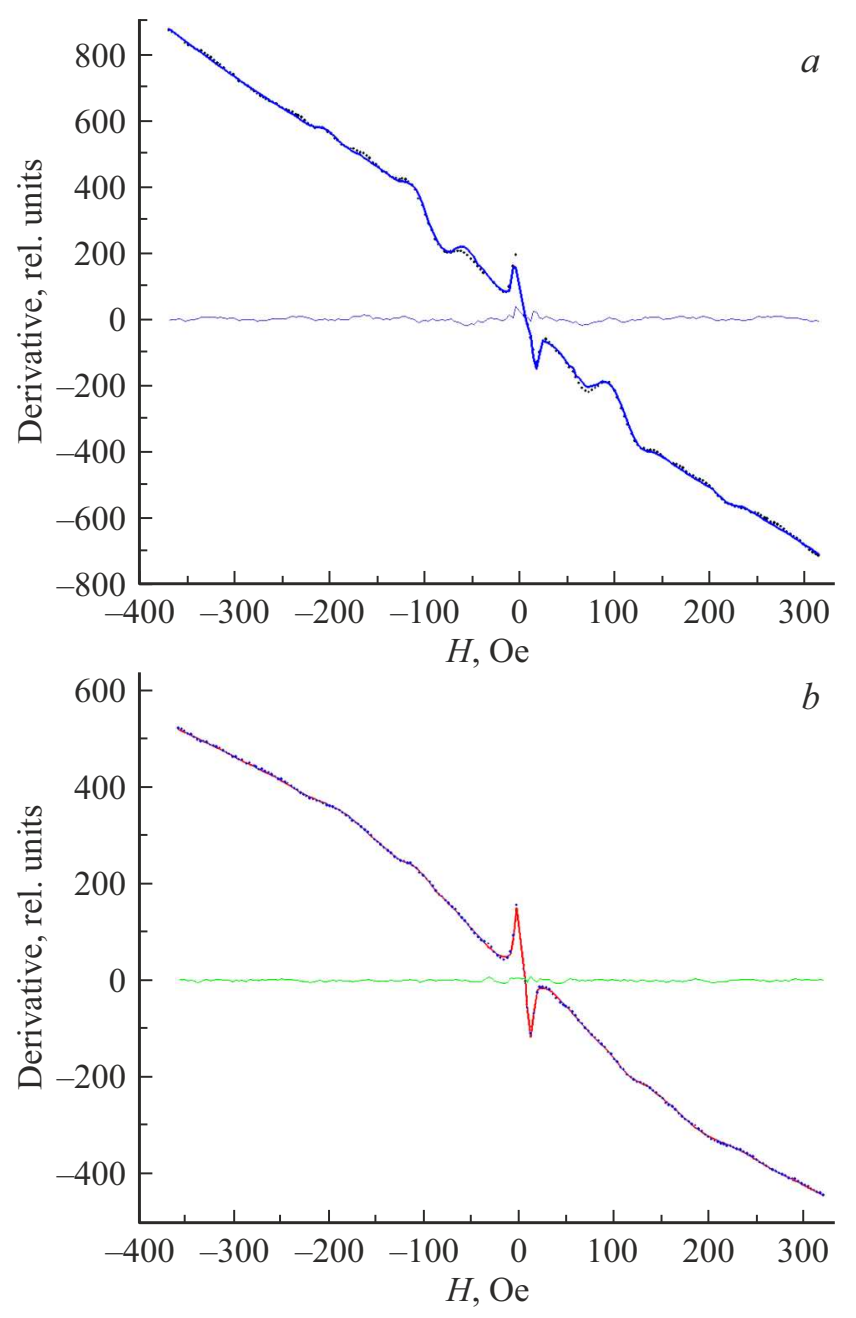

Рис. 3. Опыт 3. СФ $725 \mathrm{~nm}$, неон-20, $p<0.1$ Torr, $i=29 \mathrm{~mA}$. Горизонтальная кривая - отклонения экспериментального графика от модельной кривой („остатки“). (a) ОМРПЧ, центрированные на 10, 90, 110, 200 Ое, девиация $17 \mathrm{Gs}, 16$ проходов; $(b)$ ОМРПЧ, центрированные на 10, 110 и $200 \mathrm{Oе}$, девиация $10 \mathrm{Gs}, 28$ проходов.

$g\left(5 s_{1}{ }^{\prime}\right)=0.809$ ожидается близким $\left(-187 G_{s}\right)$. Но при этом $T_{\text {int }}=35.2 \mathrm{ns,}$, что чрезмерно, если сравнивать с кривой 1 рис. 6. Поэтому полученные данные для ширины резонанса $(7.89 \mathrm{Oe})$ и времени $T_{\text {int }}$ нельзя считать приемлемыми, несмотря на допустимость такой идентификации. Схожая ситуация сложилась у ОМРПЧ-(-200), а также с опытами 2 (рис. 2) и 4 (рис. 4) (не отраженными в табл. 1), тоже зауженными. Нельзя исключить, что причиной искажения данных по ширине ОМРПЧ-(200) может служить не учитываемый моделью ИР противоположного знака с близким смещением. Порождать такой ОМРПЧ может переход $3 p_{3}-3 d_{2}$ с длиной волны $5.67 \mu \mathrm{m}, g\left(3 d_{2}\right)=1.356$ и ожидаемым смещением 190 Gs. Сложение двух резонансов разных знаков может быть причиной обострения более узкого из них. В данном случае (при близких длинах волн) - уровня, имеющего меньший фактор 


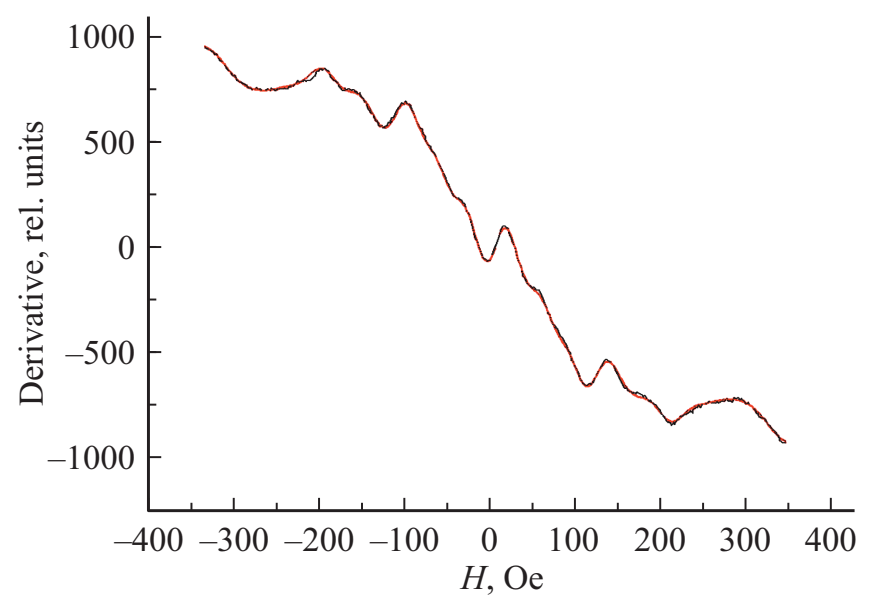

Рис. 4. Опыт 4. ОМРПЧ, центрированные на 50, 130, 200, 250 Ое. Неон-20, $p \sim 0.1$ Torr, $i=30 \mathrm{~mA}$, девиация $12 \mathrm{Gs}$, СФ ИКС-7, 5 проходов.

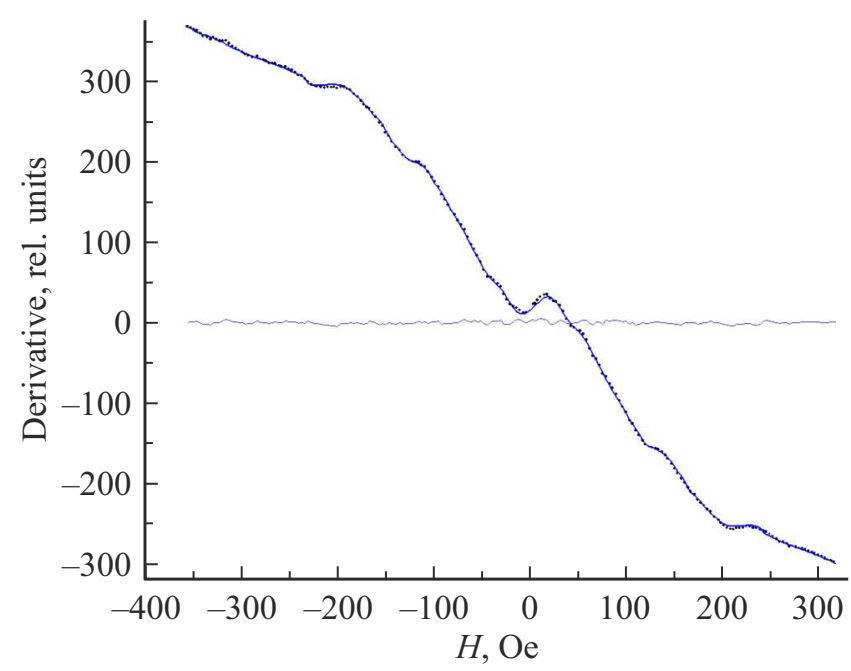

Рис. 5. Опыт 5. ОМРПЧ, центрированные на 50, 130, 200 Ое. Неон-20, $p \sim 0.1$ Torr, $i=27.6 \mathrm{~mA}$, девиация $11 \mathrm{Gs}$, СФ ИКС-7, 16 проходов. Горизонтальная кривая - отклонения экспериментального графика от модельной кривой („остатки“).

Ланде, т.е. ОМРПЧ-(-200). И только опыт 5 (рис. 5, табл. 1, строка 7) с изолированным ОМРПЧ-(-200) и увеличенным временем накопления показал более приемлемые результаты как по смещению $(-188 \mathrm{Gs})$, так и по ширине $(11.7-13.4)$ Ое. В итоге за родительский принят переход $5 d_{2}-4 p_{3}$ с длиной волны $6.06 \mu \mathrm{m}$, и $T_{\text {int }}=21.4 \mathrm{~ns}$ по верхнему пределу ширины. При этом ИС уровня $5 d_{2}$ был определен равным $237.9 \mathrm{MHz}$ по результатам измерения ИС перехода $5 d_{2}-4 p_{7}$ в [22].

Родителем ОМРПЧ-280, самого широкого резонанса опыта 1 (табл. 1 , строки 8-10), рассматривался переход $3 p_{2}-2 s_{3}$ с длиной волны $2.31 \mu \mathrm{m}$. При этом ИС уровня $3 p_{2}$ известен точно из измерений ИС линий неона $(256.43 \mathrm{MHz})$, а ИС уровня $2 s_{3}$, равный $798 \mathrm{MHz}$, установлен по данным этих измерений, что хорошо согласовалось с экспериментальными оценками ИС друших $2 s$-уровней. Однако большая ширина ОМРПЧ $(17.74 \mathrm{Oе})$ может свидетельствовать о вкладе еще и других переходов с меньшими длинами волн, центрированных на близких магнитных полях. Принимая во внимание малый контраст ОМРПЧ-280, нет смысла пытаться численными методами разрешить его структуру в опыте 1. Однако он проявил себя по-иному в других экспериментах, в частности, в опыте 2 (табл. 1 , строка 9, рис. 2) и в опыте 4 (табл. 1 , строка 10 , рис. 4 ). Численный анализ позволил выделить из его структуры ОМРПЧ-250, что привело к сужению ОМРПЧ-280 в опыте 4 (табл. 1, строка 10) и отвечало значению $T_{\text {int }}=15.08 \mathrm{~ns}$, которое и использовалось при коррекции рис. 6. Заключает перечень ИР опыта 1 визуально не видимый ИР вблизи $400 \mathrm{Oе}$, он удачно контрастирован на рис. 6 работы [8]. Здесь использование коэффициентов, корректирующих масштаб шкалы абсцисс, позволило оценить его смещение как $\sim 380 \mathrm{Oe}^{8}$

Численный анализа опыта 2 (рис. 2) выявил новые ИР в окрестности 130,50 и 250 Ое (табл. 1, строки 11 , $13,14)$. Смещение ОМРПЧ-130 составило 129.3 Ое при ширине $G_{s}=(8.25-11.0)$ Ое. В роли родителя идентифицирован переход $5 p_{5}-4 s_{3}$ с длиной волны $11.38 \mu \mathrm{m}$. В литературе нет измеренных ИС линий, которые бы включали уровни $5 p$, поэтому пришлось ориентироваться на ИС уровней $4 p(30 \mathrm{MHz})$, подтвержденные выше. Принимая во внимание стремления ИС уровней $n p \mathrm{\kappa}$ нулю по мере уменьшения их энергии, мы приняли ИС уровня $5 p_{5}$ меньшим $30 \mathrm{MHz}$ (равным $10 \mathrm{MHz}$ ). Затем, используя полученное смещение ОМПРЧ-130 и $g\left(5 p_{5}\right)=0.699$, вычислили ИС $\left(4 s_{3}\right)=137.57 \mathrm{MHz}$.

\footnotetext{
${ }^{8}$ Однако найти претендентов на порождение данного ОМРПЧ не удалось. Возможно, это слабый переход между „штрихованными“ и „нештрихованными“ уровнями неона.
}

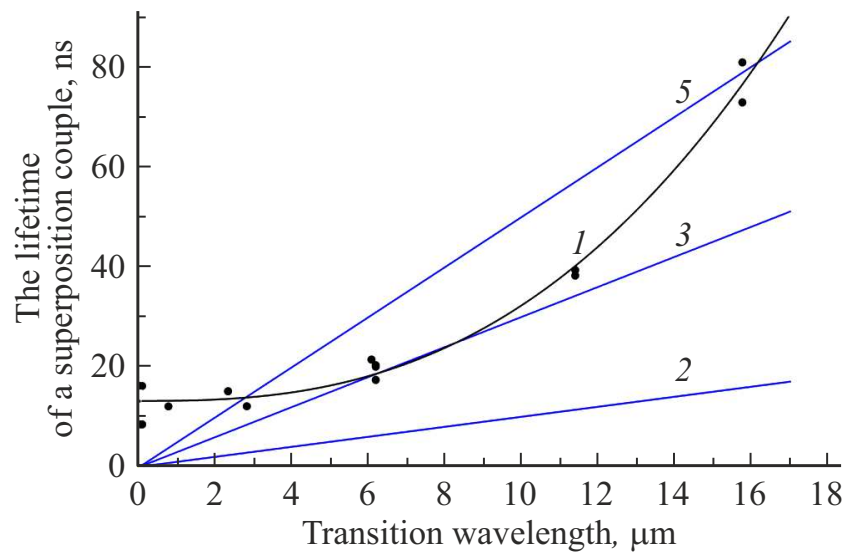

Рис. 6. Зависимость длительности интерференции атомов пары изотопов от длины волны перехода: 1 - экспериментальная зависимость, описанная функцией $T_{\mathrm{int}}=13.144+0.046 \lambda^{2.455}$; $2-5$ - времена разлета атомов изотопов на расстояние $\lambda$ в направлении, ортогональном оси пары вследствие разнонаправленного движения атомов со скоростями соответственно $\left\langle V_{t}\right\rangle, 1 / 3\left\langle V_{t}\right\rangle, 1 / 5\left\langle V_{t}\right\rangle$. 
Из разброса ширин ОМРПЧ-130 (8.25-11.0) Ое время $T_{\text {int }}$ перехода $5 p_{5}-4 s_{3}$ определили равным $(39.3-29.5) \mathrm{ns}$ с более приближенным к графику 1 рис. 6 значением 39.3 ns. ОМРПЧ-130 проявился также в опыте 4 при разбросе ширины от 7 до $9.2 \mathrm{Oе}$ и в опыте 5 с разбросом ширин от 8.17 до 8.76 Ое (табл. 1, строка 12). Последний выполнен с временем накопления сигнала, большим сравнительно с предыдущими (16 проходов против трех и пяти), поэтому его $T_{\mathrm{int}}=38.18 \mathrm{~ns}$ также использовалось при коррекции кривой 1 рис. 6.

Численный анализ малоконтрастного ОМРПЧ-50 (табл. 1, строка 13) определил его смещение равным 50 Ое. При этом была возможна аппроксимация и отрицательным ОМРПЧ с другими параметрами смещения, что ставит под сомнение надежность зарегистрированного на опыте ОМРПЧ-50 и его идентификации. Тут нужны измерения в других условиях, с большим контрастом ИР и при меньшем шаге сканирования.

В результатах численного анализа опыта 2 в пределах широкой структуры ОМРПЧ-280 был выявлен скрытый ОМРПЧ-250, центрированный на 233-239 Ое с шириной (5.26-7.46) Ое (табл. 1, строка 14). Он же проявился и в опыте 4 на 248-260 Ое с шириной (6.46-9.15) Ое (табл. 1, строка 15). В качестве родительского выступает переход $3 d_{6}-2 p_{10}$ с длиной волны $0.75 \mu \mathrm{m} \mathrm{с} \mathrm{известным} \mathrm{ИС}\left(2 p_{10}\right)=1178 \mathrm{MHz}$ и выбором ИС $\left(3 d_{6}\right)=483.6 \mathrm{MHz}$, близким к известным ИС других $3 d$-уровней, обеспечивающий максимальную ширину $9.15 \mathrm{Oe}$ и $T_{\mathrm{int}}=12.04 \mathrm{~ns}$ в согласии с кривой 1 рис. 6.

Следующий опыт 3 (табл.1, строки 16, 17), выполненный при близких условиях по давлению и току разряда, но при другой спектральной фильтрации излучения, выявил ОМРПЧ вблизи 10 Ое. При использовании светофильтра (СФ) ИКС-7 кривая была схожей с кривой рис. 2 в части формы широкой структуры вблизи нуля магнитного поля. Однако при установке СФ $725 \mathrm{~nm}$ она сменилась на более узкую и другого знака. Перекачка смеси дополнила опыт структурой вблизи $100 \mathrm{Oe}$ (рис. $3, a$ ), похожей на дисперсионный контур, копирующей такую же парную структуру графика опыта 1. Поэтому не вызывает сомнения, что данные резонансы порождены ИК переходами между теми же уровнями. Однако эти опыты выполнены без ИК светофильтра, поэтому нельзя гарантировать, что с интерференционным светофильтром также регистрируется излучение слабо поглощающих переходов, и, действительно, можно ли принять коэффициент коррекции масштаба магнитной шкалы $k_{m}$ отвечающим среднему магнитному полю (0.954). В данном случае можно определить $k_{m}$, используя в качестве реперного значения смещения резонансов, зарегистрированные в опыте 1. Конкретно было использовано смещение более контрастного ОМРПЧ-90. Полученные значения параметра $k_{m} \approx 0.96$ оказались лишь немного большими, чем получаемые с ИК фильтрами. По-видимому, это связано с тем, что из пяти линий, попадающих в полосу пропускания СФ-725, две линии с уровней $2 p$ на уровни $1 s_{2}$ и $1 s_{4}$ настолько сильно поглощающие, что сигнал определяется лишь тремя слабыми переходами, для которых кювета прозрачна и магнитное поле близко к среднему. Результаты аппроксимации ОМРПЧ данного опыта приведены на рис. $3, a$ и в табл. 1 (строка 16). Смещение нового ИР определилось равным $11.3 \mathrm{Oe}$ с $G_{s}=2.37 \mathrm{Oe}$. В качестве родительского выступает переход $5 s_{2}-5 p_{1} \mathrm{c}$ $\lambda=15.76 \mu \mathrm{m}$, с оценкой ИС $\left(5 s_{2}\right)=30.26 \mathrm{MHz}$, установленной при ИС $\left(5 p_{1}\right)=10 \mathrm{MHz}$, ожидаемой для $p$-уровней. При этом получено значение $T_{\mathrm{int}}=72.8 \mathrm{~ns}$, максимальное для эмпирической зависимости $T_{\text {int }}$ от длины волны.

Помимо этого в данной серии имелся опыт, где визуально просматривался только узкий ОМРПЧ вблизи $H=0$. Однако благодаря большему времени накопления сигнала (28 проходов вместо 16) численная обработка выявила в нем присутствие еще двух ИР ОМРПЧ0-(-110) и ОМРПЧ-(-200). Отличие этого опыта состоит также в меньшей девиации магнитного поля (10 Gs вместо $17 \mathrm{Gs}$ ), что позволяет оценить вклад девиации в ширину ОМРПЧ-(-10). Поэтому был проведен и его анализ при использовании в качестве репера смещение ОМРПЧ-(-110), определенное в опыте 1. При этом использовались два подхода: с калибровкой шкалы по результатам опыта 3, рис. 3, $a$ (по ОМРПЧ-90), и по ОМРПЧ-(-110). Результаты получились близкими и демонстрируются на рис. $3, b$ (табл. 1 , строка 17). При этом возросли параметры $k_{m}$ и $k_{p}(0.988$ и 0.999$)$, и это сказалось на смещении и ширине ОМРПЧ-(-10). Основное отличие от результатов на рис. $3, a$ в том, что смещение ОМРПЧ-(-10) оказалась несколько меньшим (7.6 Ое), но укладывающимся в ожидаемое смещение при коррекции оценки ИС $\left(5 s_{2}\right)=30 \mathrm{MHz}$ к значению $24 \mathrm{MHz}$, а интересующая нас ширина определилась равной $2.14 \mathrm{Oe}$ против $2.37 \mathrm{Oe}$ (рис. $3, a$ ). Это приводит к некоторому увеличению $T_{\mathrm{int}}=80.8 \mathrm{~ns}$, но принципиального влияния на ход кривой 1 рис. 6 не оказывает. ${ }^{9}$

Таким образом, экспериментальная кривая 1 рис. 6, равно как и данные по ИС высоковозбужденных уровней неона, полученные в результате идентификации представленных ОМРПЧ, будут полезны при дальнейшем исследовании ИР, порождаемых ИК переходами. Их уточнение поможет идентификации новых ОМРПЧ, и прояснению динамики спектроскопических переходов при анализе полей ближней зоны излучения атомов.

И в завершение для сравнения с $T_{\text {int }}$ переходов в УФ области спектра на кривую 1 рис. 6 помещены результаты аппроксимации квадратурного ОМРПЧ-1330 (табл. 1 , строка 18), порождаемого переходом $2 s_{4}-{ }^{1} S_{0}$ с длиной волны $63 \mathrm{~nm}$ из работы [10] (точки у оси

\footnotetext{
${ }^{9}$ Ширина ОМРПЧ-(-110), в этом опыте изолированного, определилась равной $8.2 \mathrm{Oe}$, что приводит к значению $T_{\mathrm{int}}=20.0 \mathrm{~ns}$ (табл. 1 , строка 5), согласующемуся с кривой 1 рис. 6 . А для ОМРПЧ-(-200) ширина определилась зауженной $(11.5 \mathrm{Oe})$ сравнительно с опытом 5 , табл. 1, строка 7 (13.4Ое), что свойственно этому резонансу (см. выше).
} 
ординат). Они, как и вся кривая 1 , не демонстрируют стремления к нулю оси ординат, обусловленного сокращением времени существования КСП атомов при росте частоты перехода.

\section{Обсуждение результатов}

Одним из результатов работы стало обнаружение ИР со знаком амплитуд, противоположным знакам ОМРПЧ, порождаемых переходами в видимом диапазоне спектра. Отрицательные ИС были выявлены у трех ИК переходов: $5 s_{1}{ }^{\prime}-4 p_{1} \quad(\lambda=5.85 \mu \mathrm{m}$, ИС $\left.=-211.8 \mathrm{MHz}, \quad H_{r}=-187 \mathrm{Gs}\right), \quad 5 d_{5}-4 p_{3}$ $\left(\lambda=6.17 \mu \mathrm{m}, \quad\right.$ ИС $\left.=-213.04 \mathrm{MHz}, \quad H_{r}=-110 \mathrm{Gs}\right)$, $5 s_{2}-5 p_{1} \quad(\lambda=15.76 \mu \mathrm{m}, \quad$ ИС $=-24.36 \mathrm{MHz}$, $\left.H_{r}=-7.8 \mathrm{Gs}\right)$. Помимо этого было обнаружено, что среди ИК переходов, измеренных в работе [22], имеются еще три перехода с отрицательными ИС. Это переходы $5 d_{2}-4 p_{7}(\lambda=5.3264 \mu \mathrm{m}$, ИС $=-207.9 \mathrm{MHz}), 5 d_{1}{ }^{\prime \prime}-4 p_{6}$ $(\lambda=5.3258 \mu \mathrm{m}, \quad$ ИС $=-207.9 \mathrm{MHz}) \quad$ и $\quad 5 s_{1}{ }^{\prime \prime \prime \prime}-4 p_{5}$ $(\lambda=5.17 \mu \mathrm{m}$, ИС $=-211.8 \mathrm{MHz})$, чьи ИС были ошибочно определены как положительные. Эти переходы не имеют невырожденного уровня и не могут порождать ОМРПЧ, но позволили уточнить экстраполяционные оценки ИС высоковозбужденных $d$-уровней.

Результаты показывают, что идентификация ОМРПЧ, порождаемых ИК переходами, несмотря на недостаток опытных данных о параметрах высоковозбужденных уровней, возможна. Этому помогает экстраполяция опытных данных о ИС уровней на более высоко возбужденные.

Важным результатом работы стало построение эмпирической зависимости времени интерференции полей пары когерентно связанных изотопов (времени жизни парной когерентности) от длины волны перехода, представленной кривой 1 рис. 6 . Это позволяет задействовать при идентификации ОМРПЧ не только их смещения в шкале магнитного поля, но и сравнение их ширин с ожидаемыми ширинами, задаваемыми длиной волны переходов. В этом практическая польза графика с точки зрения идентификации ОМРПЧ. Но остается вопрос, какую физическую информацию о суперпозиционном состоянии пары когерентно связанных атомов эта зависимость отображает, и насколько она подвержена искажению в ансамбле движущихся атомов.

Еще в работе Дикке [14] было показано, что межатомные квантовые корреляции пространственно близких возбужденных неподвижных атомов могут приводить не только к ускорению их совместного распада, названного им сверхизлучением, но и к его замедлению, впоследствии названному субизлучением. В работе [13] отмечено, что корреляции могут носить как квантовый, так и классический характер. В переложении на „классические“ процессы, протекающие в обобщенной ближней зоне, сверхизлучение отвечает синфазной синхронизации движения зарядов диполей. В этом случае заряды складываются, суммарный ток пары диполей и скорость излучения возрастают, а время $T_{\text {int }}$ уменьшается. Субизлучение отвечает противофазной синхронизации движения зарядов, когда заряды вычитаются, суммарный ток и скорость излучения падают, и время $T_{\text {int }}$ должно возрастать. В исследуемом нами случае квадратурной синхронизации со сдвигом фазы $\pi / 2$ суммарный колеблющийся заряд и ток диполей не меняются, и время $T_{\text {int }}$ в пределе равно времени нахождения одиночного атома в суперпозиционном состоянии перехода между стационарными состояниями. Фактически оно, конечно, меньше, поскольку определяется временем совместного существования полей двух таких атомов, различающимся от пары к паре в связи с разной задержкой поля вынужденного относительно вынуждающего.

Помимо этого ход данной кривой определяется также зависимостью времени перекрытия ближних зон изотопов от относительных скоростей атомов пары. Необходимо было выяснить, что в итоге определяет длительность интерференции полей: время жизни суперпозиционного состояния $T_{\text {int }}$ или длительность времени перекрытия ближних зон, обусловленная движением атомов пары. Здесь есть два механизма влияния движения.

В первом случае речь идет о проекциях скоростей, лежащих в плоскости, ортогональной магнитному полю, и обеспечивающих доплеровский сдвиг $k \Delta v$, компенсирующий отстройку магнитного поля от центра резонанса:

$$
k \Delta v=\mu_{B} g\left(|H|-H_{r}\right),
$$

при переходе к скоростям

$$
\Delta v=\mu_{B} g\left(|H|-H_{r}\right) \lambda / 2 \pi .
$$

Из (11) видно, что на пробег расстояния $\lambda$, равного области, отвечающей максимальным амплитудам КСП изотопов, затрачивается время $\Delta t$, не зависящее от $\lambda$ :

$$
\Delta t=\lambda / \Delta v=2 \pi /\left[\mu_{B} g\left(|H|-H_{r}\right)\right] .
$$

При отстройке на ширину $G_{s}$ резонансов ОМРПЧ, введенную в (8) и связанную с $T_{\text {int }}$ соотношением $G_{s}=1 /\left(4 \pi \mu_{B} g_{m} T_{\text {int }}\right)$, получаем, при $\mu_{B}=1.404 \mathrm{MHz} / \mathrm{Gs}$ оценку (в ns)

$$
\Delta t=637.2 /\left(g_{m} G_{s}\right),
$$

в 2.8 раза превышающую оценку $T_{\mathrm{int}}$, следующую из (9). Поэтому время пересечения ближних зон изотопов не может служить фактором, ограничивающим $T_{\text {int }}$ сверху.

Вторая ситуация реализуется при рассмотрении проекций скоростей атомов, направленных ортогонально линии, соединяющей атомы изотопической пары, т.е. вдоль магнитного поля. Здесь относительная скорость атомов изотопов определяется их тепловой скоростью, которая может превышать величину, задаваемую (11), и нет прямой зависимости от напряженности магнитного поля. Однако входит длина волны как мера наклона линии, соединяющей атомы изотопов, относительно плоскости, ортогональной направлению магнитного поля и 
ее удлинения. Поэтому здесь можно ожидать действие различия поперечных скоростей изотопов, ограничивающее $T_{\text {int. }}$. Но $\Delta t \sim \lambda / V_{t}$, и это линейная функция от длины волны, обратно пропорциональная квадратному корню из температуры газа.

При скоростях изотопов пары $\left\langle V_{t}\right\rangle \sim 0.5 \cdot 10^{5} \mathrm{~cm} / \mathrm{s}$, равных средней скорости атомов газа неона в нормальных условиях, но противоположно направленных, $\Delta t$ представлено на рис. 6 кривой 2. Эта кривая расположена ниже кривой 1. При такой большой относительной разности поперечных скоростей изотопов образование когерентных пар с большими временами $T_{\text {int }}$, демонстрируемыми кривой 1 рис. 6 , невозможно. Такая возможность появляется для пар с меньшей поперечной скоростью изотопов. На рис. 6 кривая 3 отвечает скорости в 3 раза, а кривая 5 - в пять раз меньшей, чем для кривой 2 . Но когда $\Delta t$ в стремлении к нулю при уменьшении $\lambda$ пересекает кривую 1 , это должно приводить к уменьшению $T_{\text {int }}$ на этом отрезке шкалы абсцисс, тогда как на опыте наблюдается выход на стационарное значение $\sim 13.5 \mathrm{~ns}$.

Выше обсуждалось, что рост $T_{\text {int }}$ можно толковать как переход пары изотопов в субизлучательное состояние с замедлением радиационного распада атомов, но это удивительно, поскольку здесь мы исследуем ОМРПЧ c квадратурной синхронизацией колебаний, поэтому уменьшение суммарного заряда и тока диполей при противофазной синхронизации, приводящее к эффекту субизлучения, должно отсутствовать. К тому же противофазную синхронизацию нельзя обеспечить с помощью КСП ближней зоны излучения, эффект запаздывания для которых отсутствует, что способствует синфазной синхронизации и препятствует противофазной. Поэтому возникает необходимость привлечения волновых полей формирующегося фотона, которые до момента накопления энергии $\hbar \omega$ тоже по сути дела поля реактивные, поскольку они не способны к передаче энергии, но могут обеспечить синхронизацию колебаний. Однако в случае квадратурных ОМРПЧ сдвиг фазы $\pi / 2$ обусловлен инициализацией вынужденного испускания изотопического партнера и не зависит от расстояния между ними. Во всех случаях будет регистрироваться квадратурная форма ОМРПЧ (пик в производной). Но в случае противофазной синхронизации производная должна иметь дисперсионно подобную форму [10], чего не наблюдается.

Это свидетельствует о наличии эффекта, природа которого непонятна. Возникает предположение, что стремление кривой 1 к ненулевому пределу при укорочении длины волны не связано с субизлучением, а обусловлено удлинением времени некогерентного отклика окружающих возбужденных атомов на поле интерференции когерентной пары. То есть дезактивацией близких возбужденных атомов, растущей с ростом вероятности перехода пропорционально кубу частоты и растянутой во времени в связи с последовательным подключением к процессу все новых слоев атомов. Это время определяется скоростью распространения процесса дезактивации, зависящей от вероятности перехода, длины волны и размера кюветы.

Озадачивает и нелинейность увеличения времени жизни суперпозиционной пары с увеличением длины волны, которое выше определенных длин волн должно приобрести линейный характер. Возможно, эти длины волн в данных опытах не достигнуты. А для уменьшения длительности нелинейного отклика следует уменьшать концентрацию возбужденных атомов или диаметр кюветы, что, конечно, будет уменьшать амплитуду ИР. Для прояснения этих возможностей требуется разработка теоретической модели процесса и дополнительные опыты с ОМРПЧ, порождаемыми как ИК, так и УФ переходами.

\section{Заключение}

Таким образом, полученные результаты заложили базу для идентификации новых ИР с использованием экспериментальной зависимости ширин квадратурных ОМРПЧ от длины волны порождающих их переходов, но одновременно поставили вопросы о причинах ее формирования.

В работе был обнаружен выход на плато времени пребывания в суперпозиционном состояний в области малых длин волн с временем намного большим, чем ожидается для больших частот переходов. Это было связано с увеличением длительности отклика среды на поле интерференции изотопической пары. Вероятность дезактивации возбужденных атомов окружения растет кубично с ростом частоты переходов и зависит от концентрации возбужденных атомов и размера кюветы. Но с чем могут быть связаны большие времена $T_{\text {int }}$ в области длинноволновых ИК-переходов? Здесь и частоты малы и населенность высоковозбужденных уровней мала. В связи с этим возникают следующие вопросы.

Чем определяются измеренные на опыте конечные времена $T_{\text {int }}$ пребывания пары изотопов в когерентно связанном состоянии, из чего следует конечность времен перехода атомов из одного стационарного состояния в другое?

Насколько они могут быть связаны с предсказанным Гейзенбергом протяженным, а не скачкообразным характером атомных переходов ${ }^{10}$

Какую роль в выявлении этой протяженности играют реактивные поля ближней зоны излучения атома?

Эти вопросы выходят за рамки данной статьи, и будут рассмотрены позднее.

\footnotetext{
10 Вопросы динамики спектроскопических переходов в представлении Гейзенберга рассмотрены в разд. 4.4 монографии [24].
} 


\section{Финансирование работы}

Работа выполнена в рамках приоритетных исследований СО РАН (программа II.10.2).

\section{Благодарности}

Автор благодарит В.А. Сорокина, в соавторстве с которым были получены результаты по идентификации ИР в работе [8], А.М. Шалагина и С.Л. Микерина за поддержку работы, Л.В. Ильичева за замечания, сделанные при ознакомлении с рукописью статьи.

\section{Конфликт интересов}

Автор заявляет, что у него нет конфликта интересов.

\section{Список литературы}

[1] E.G. Saprykin, V.A. Sorokin. In: IVth Int. Symposium Modern Problems of Laser Physics (Novosibirsk, 2004), p. 240.

[2] Э.Г. Сапрыкин, В.А. Сорокин. В сб.: Tруды IV международной конференции Проблемы фундаментальной оптики (Санкт-Петербург, 2006), с. 173.

[3] М.П. Чайка. Интерференция вырожсенных состояний (Л.: Изд-во ЛГУ, 1975).

[4] Е.Б. Александров, Г.И. Хвостенко, М.П. Чайка. Интерференция атомных состояний (М.: Наука, 1991).

[5] Э.Г. Сапрыкин, В.А. Сорокин. Опт. и спектр., 109, 573 (2010).

[6] Э.Г. Сапрыкин, В.А. Сорокин, А.М. Шалагин. ЖЭТФ, 143, $622(2013)$

[7] V.A. Sorokin. In: Sixth Int. Simposium Modern Problem of Laser Physics (Novosibirsk, 2013), p. 241-242.

[8] Э.Г. Сапрыкин, В.А. Сорокин. Опт. и спектр., 117, 18 (2014).

[9] Э.Г. Сапрыкин, В.А. Сорокин, А.М. Шалагин. Квант. электрон., 48, 827 (2015).

[10] Э.Г. Сапрыкин. ЖЭТФ, 149, 251 (2016).

[11] Э.Г. Сапрыкин. Опт. и спектр., 122, 568 (2017).

[12] Р. Фейнман, Р. Лейтон., М. Сэндс. Фейнмановские лекции по физике. Т. 5. Электричество и магнетизм (М.: Мир, 1977).

[13] Л.В. Ильичев. ЖЭТФ, 131, 30 (2007).

[14] R.H. Dicke. Phys. Rev., 93, 99 (1954).

[15] А.А. Макаров, В.С. Летохов. ЖЭТФ., 124, 766 (2003).

[16] W. B. Westerveld, J. van Eck. J. Phys. B., 12, 377 (1979).

[17] K.S.E. Eikema, W. Ubachs, W. Hogervorst. Phys. Rev. A., 49, 803 (1994).

[18] H. Shober. Pys. Zs., 40, 77 (1939).

[19] Э.Г. Сапрыкин. Опт. и спектр., 120, 222 (2016).

[20] Э.Г. Сапрыкин. Опт. и спектр., 127, 179 (2019).

[21] Э.Г. Сапрыкин. Опт. и спектр., 123, 285 (2017).

[22] J.C. Keller, J.F. Lesprit. Physica., 64, 202 (1973).

[23] П.Ф. Груздев. Вероятности переходов и радиащионные времена жсизни уровней атомов и ионов (М.: Энергоатомиздат, 1990).

[24] J.D. Macomber. The Dynamics of Spectroscopic Transitions (New York-London-Sydney-Toronto: John Wiley and Sons, 1976). [Дж. Д. Макомбер. Динамика спектроскопических переходов (М.: Мир, 1979)]. 